\title{
Paragonimus sp. en cangrejos y sensibilización de la comunidad educativa hacia los ecosistemas acuáticos de La Miel y La Clara, Caldas, Antioquia
}

\author{
Sandra Milena Arias ${ }^{1,2}$, Lina Marcela Salazar ${ }^{1,2}$, Eudoro Casas $^{1}$, Alexandra Henao $^{3}$, \\ Luz Elena Velásquez ${ }^{1,2}$ \\ 1 Grupo Microbiología Ambiental, Escuela de Microbiología, Universidad de Antioquia, Medellín, Colombia \\ 2 Programa de Estudio y Control de Enfermedades Tropicales, PECET, Facultad de Medicina, Universidad de \\ Antioquia, Medellín, Colombia \\ ${ }^{3}$ Grupo Diverser, Facultad de Educación, Universidad de Antioquia, Medellín, Colombia
}

Introducción. Estudios previos demostraron la presencia de Paragonimus sp. en cangrejos de tres municipios en la cuenca del río Medellín. Para diseñar un plan para el control y la prevención de la paragonimosis, es necesario determinar la presencia del parásito en la parte alta del río, con la participación de la comunidad educativa, para dirigir su atención hacia el recurso hídrico.

Objetivo. Establecer la presencia de metacercarias de Paragonimus $\mathrm{sp}$. en cangrejos de las quebradas La Miel y La Clara, y sensibilizar a la comunidad educativa hacia el manejo adecuado de sus ecosistemas acuáticos.

Materiales y métodos. En 2007 y 2008 se recolectaron cangrejos de quebradas de las veredas La Miel y La Clara. En el laboratorio se relajaron y sacrificaron para buscar metacercarias de Paragonimus $\mathrm{sp}$. y determinar la especie. Con estudiantes de instituciones educativas de ambas veredas, se llevaron a cabo cinco talleres lúdicos, para mejorar la percepción de su relación con sus quebradas.

Resultados. Se capturaron nueve cangrejos Hypolobocera bouvieri. En La Miel se halló uno con Paragonimus sp. En La Clara se encontró, además, Aroapyrgus colombiensis, molusco huésped del parásito. Con los estudiantes se estableció que los ecosistemas acuáticos ejercen una gran atracción sobre ellos; sin embargo, desconocen los riesgos y no sostienen una relación responsable.

Conclusiones. Se señala la presencia de focos de Paragonimus sp. en la parte alta del río Medellín; por lo tanto, el municipio de Caldas debe incluirse en los programas de prevención y control. La población estudiantil requiere nuevos programas educativos para mejorar la interacción con los ecosistemas acuáticos y disminuir el deterioro de su salud.

Palabras claves: Paragonimus sp., ambiente acuático, educación, salud.

Paragonimus sp. in crabs and awareness of the educational community to aquatic ecosystems in La Miel and La Clara, Caldas, Antioquia

Introduction. Previous research has demonstrated the presence of Paragonimus spp. in crabs of three municipalities located in the Medellín river basin. To design a plan for the control and prevention of paragonimosis, parasite presence must be demonstrated in the upper area of the river, and an awareness campaign of its risks must involve the participation of the educational community in focusing attention on the local aquatic resources.

Objective. The presence of Paragonimus spp. metarcercariae was established in freshwater crabs from La Miel and La Clara streams, and an awareness campaign created public awareness to management of the aquatic ecosystems for risk reduction.

Materials and methods. Nine crabs were collected from streams of La Miel and La Clara villages in 2007 and 2008. The crabs were indentified, sacrificed in the laboratory and examined for presence of Paragonimus spp. metacercariae. Five workshops were conducted with students of educational institutions of the two villages to improve the student perception of paragonimosis risks associated with the local streams.

Results. The crabs were identified as Hypolobocera bouvieri. One crab was found with Paragonimus spp. in La Miel. A mollusk, Aroapyrgus colombiensis, another host of the parasite was found in La Clara. That aquatic ecosystems are a great attraction for the local was well established.

Conclusions. Foci of Paragonimus spp. flatworms are present in the upper area of Medellín river which includes Caldas. However, local school children were uninformed about the risk of paragonimosis associated with the local aquatic ecosystem. Therefore this province must be included in programs for prevention and control of paragonimosis. The prevalence of this disease recommends educational programs that promote awareness of local aquatic ecosystems and their parasitic inhabitants.

Key words: Paragonimus spp., aquatic environment, education, health. 
Antioquia es un departamento de Colombia rico en ecosistemas acuáticos, en el que habitan diversas especies de cangrejos. Estos crustáceos son de interés epidemiológico como huéspedes intermediarios de los trematodos digéneos Paragonimus spp., que causan deterioro en la salud de las personas, animales domésticos y silvestres, pues generan pérdidas económicas significativas en países tropicales y subtropicales de Asia, África y América $(1,2)$.

En su ciclo de vida, Paragonimus spp. requiere de un molusco como primer huésped intermediario, un crustáceo como segundo huésped intermediario y un mamífero como huésped definitivo $(3,4)$. En relación con sus huéspedes, este trematodo ha mostrado mayor especificidad por los moluscos, que en Suramérica corresponden a caracoles del género Aroapyrgus (Prosobranchia: Hydrobiidae), muy abundantes en los ecosistemas acuáticos colombianos $(5,6)$.

En lo que respecta a los huéspedes crustáceos, en Antioquia se ha registrado la presencia de varias especies asignadas a los géneros Hypolobocera y Strengeriana de la familia Pseudothelphusidae (7). Asimismo, en este departamento se ha señalado a las personas y a Didelphis marsupialis como huéspedes definitivos del digéneo (8).

Por lo general, los cangrejos se encuentran en ecosistemas acuáticos de los sectores rurales; sin embargo, los afluentes del río Medellín en la zona urbana de este municipio, de Envigado y de Girardota, han demostrado ser hábitats ricos en fauna de cangrejos parasitada con el digéneo (7), lo que constituye un factor de riesgo muy grande para los pobladores asentados en las orillas de los ecosistemas acuáticos que conforman la cuenca del río Medellín, lo que conlleva al planteamiento de estrategias para la prevención y el control de esta parasitosis.

En lo que respecta a las campañas para el control y la prevención de diversas parasitosis, se logra un mayor éxito cuando se involucra a la comunidad en el diseño y en el desarrollo de las mismas, al establecer en las instituciones educativas los

\section{Correspondencia:}

Luz Elena Velásquez, Programa de Estudio y Control de Enfermedades Tropicales, PECET, Facultad de Medicina, Universidad de Antioquia, Calle 62 № 52-59, torre 2, laboratorio 730, Medellín, Colombia.

Teléfono: (574) 219 6514; fax: (574) 2196511

luzelena333@yahoo.com

Recibido: 28/10/10; aceptado:22/02/11 centros de actividades. En Nepal, durante 1998, se puso en marcha un curso para docentes, con el fin de prevenir la infestación por el digéneo Schistosoma mansoni, el cual incluyó la enseñanza de comportamientos saludables a los estudiantes y el suministro del antiparasitario mediante jornadas de desparasitación en las escuelas. La evaluación realizada en el 2000 demostró que el programa redujo en $80 \%$ el número de personas infectadas, por lo que se unieron a la iniciativa países como Burkina Faso, Camerún, Ghana, Kenya, Malawi, Malí, Níger, Nigeria, República Unida de Tanzania y Zambia (9).

De otro lado, la esquistosomosis se logró controlar y erradicar en China y Japón, respectivamente, por medio de intervenciones en los que participó activamente la comunidad $(10,11)$.

Por lo tanto, la presente investigación se propuso verificar la presencia de Paragonimus spp. en la cabecera del río Medellín, involucrando a la comunidad educativa (docentes y estudiantes), con el fin de sensibilizarla hacia el uso adecuado del recurso hídrico, como preámbulo a una campaña de prevención y control de la paragonimosis.

\section{Materiales y métodos \\ Descripción del área de estudio}

Para esta investigación se seleccionaron las veredas La Miel y La Clara del municipio de Caldas (Antioquia) porque sus quebradas (La Miel y La Clara) junto con La Valeria dan origen al río Medellín (12). El municipio ocupa el extremo sur del valle de Aburrá y hace parte de la denominada área metropolitana (13).

En la vereda La Miel, sobre los 1.872 msnm, se contactó la institución educativa Monseñor Pedro Luis Álvarez, donde estudian 155 alumnos en los grados de preescolar a quinto de primaria (14).

En la vereda La Clara, ubicada a una altitud entre 1.798 msnm y 2.017 msnm, se trabajó con la escuela rural dirigida por la Institución Educativa Ciro Mendía, con 69 niños en los grados de preescolar y básica primaria (14).

En primera instancia, el proyecto fue presentado a las autoridades municipales las cuales aprobaron su realización.

\section{Procedimientos}

En los cauces de las quebradas La Miel y La Clara y en sus proximidades, se hizo búsqueda activa de cangrejos y moluscos dulciacuícolas. En los sitios 
de captura se estimaron las coordenadas con un sistema de localización global (Global Positioning System, GPS) marca Etrex®.

Los cangrejos y moluscos recolectados se transportaron vivos e individualizados en tarros con agua fresca del lugar. Se llevaron al laboratorio de Malacología Médica y Trematodos del Programa de Estudio y Control de Enfermedades Tropicales (PECET, Universidad de Antioquia), donde se instalaron en acuarios.

Para buscar las metacercarias, los cangrejos se sacrificaron sumergiéndolos en agua a $2^{\circ} \mathrm{C}$ durante 10 minutos. Luego se disecaron bajo estereomicroscopio y se retiraron las formas larvarias de los parásitos. Éstas se fijaron en formalina acéticaalcohol ácido durante 24 horas, y se conservaron en alcohol al $70 \%$; se colorearon con carmín de Meyer, se aclararon con metilsalicilato y se montaron en bálsamo de Canadá. Con un micrómetro ocular, se estimaron las siguientes dimensiones: largo y ancho del cuerpo, los diámetros de la ventosa oral y del acetábulo, y la distancia entre éstas. Las placas permanentes se depositaron en la Colección Colombiana de Helmintos CCH116. Se tomaron registros fotográficos.

Para hacer la determinación taxonómica de los cangrejos, se tuvo en cuenta la morfología de los gonópodos y del tercer maxilípedo $(15,16)$, los cuales se fotografiaron.

Los moluscos se instalaron en acuarios adecuados con agua sin cloro y aireación artificial, a la temperatura y el $\mathrm{pH}$ del hábitat natural. Se alimentaron con lechuga fresca. Algunos ejemplares se sacrificaron para su determinación taxonómica y los demás se fijaron en alcohol al $70 \%$ y se depositaron en la colección de moluscos vectores VHET 37 de la Universidad de Antioquia. Se tomaron registros fotográficos.

\section{Intervención educativa}

En cada institución educativa se informó a los padres de familia sobre el proyecto, y se pidió consentimiento para que sus hijos participaran en él.

Se inició con una encuesta en la que se identificó una inadecuada relación entre el estudiantado y las fuentes hídricas de su vereda; por esto, se diseñaron cinco talleres que se implementaron en la comunidad educativa, para mejorar la percepción de los niños sobre los ecosistemas acuáticos.

Con el primer taller se pretendió indagar sobre los conocimientos de la población estudiantil sobre su contexto geográfico. Para ello, se utilizó un plano de la vereda con algunos lugares de referencia (escuela, puente, quebrada); en el plano, los niños debían localizar el sitio de su vivienda y, sobre él, pegar un trocito de balso en representación de ésta; además, ubicar círculos verdes de papel en donde ellos consideraban que los árboles eran más abundantes. También, se les solicitó dibujar las fuentes hídricas más próximas a su casa y las más frecuentadas para fines recreativos y laborales. Para esta actividad, cada grado de escolaridad dispuso de un plano.

Para el segundo taller se exploró la percepción de la población estudiantil sobre el estado ambiental de las fuentes hídricas, dibujando sobre el plano anterior las quebradas limpias con color azul y, las sucias, con color rojo, exponiendo, además, las razones para ello, al igual que se indagó sobre su concepto de naturaleza.

Con el propósito de mejorar la percepción y el conocimiento del estudiantado y las maestras sobre los organismos dulciacuícolas, durante el tercer taller se montó un acuario en dos etapas; en la primera, se instalaron los componentes abióticos (agua, aire, arenón y piedras) y los estudiantes elaboraron un manual para su manejo y cuidado. En la segunda etapa, se realizó una salida de campo con los estudiantes para capturar los organismos e introducirlos en el acuario.

En el cuarto taller se indagó sobre la importancia ecológica que la comunidad escolar le confiere a los sitios de su vereda, para lo cual se organizaron en grupos; cada uno eligió el lugar que consideraba de mayor impacto ambiental y lo recrearon por medio de una dramatización; a su vez, las facilitadoras asumieron el papel de turistas y pasaron por cada grupo haciendo preguntas que permitieron conocer el valor que los niños le asignaban a la fauna y a la flora de estas zonas, y la relación que ellos construyeron con ese lugar.

El quinto taller se realizó con el fin de conocer las problemáticas ambientales identificadas por la población estudiantil, las cuales expresaron en conjunto. Posteriormente, por equipos, elaboraron un afiche con las causas y las alternativas de solución e invitando a la comunidad a participar en éstas. Al terminar la actividad, se divulgó y se reflexionó sobre los aprendizajes adquiridos.

\section{Resultados}

Los macroorganismos recolectados durante la búsqueda de huéspedes intermediarios de 
Paragonimus spp. en los ecosistemas acuáticos de las veredas La Miel y La Clara y el número de especímenes, se presentan en el cuadro 1, donde se observa que en la vereda La Clara, aunque no se halló el digéneo, se encuentran todos los huéspedes involucrados en el ciclo de vida del parásito, incluyendo los moluscos Aroapyrgus colombiensis, de los que algunos ejemplares reposan en la colección VHET 37, bajo el código 946 (figura 1); en la vereda La Miel, uno de los cangrejos recolectados presentó metacercarias de Paragonimus spp. en las coordenadas ${\mathrm{N} 06^{\circ}}^{\circ} 5^{\prime}$ 54.8" W075 $07^{\prime}$ 08.6", a una elevación de 1.911 msnm.

Los nueve cangrejos capturados fueron clasificados como Hypolobocera bouvieri de la familia Pseudothelphusidae (figura 2). Los especímenes se depositaron en la colección del Museo Universitario de la Universidad de Antioquia, bajo los códigos MUA-ICR 103, 189 y 190.
Del cangrejo parasitado se obtuvieron nueve larvas de Paragonimus spp., las cuales presentaron cuerpo color rosa, ventosa oral más pequeña que el acetábulo, seguida de una faringe corta que se bifurca en dos ciegos intestinales sinuosos, laterales y proyectados hasta el extremo posterior, acetábulo ecuatorial y sistema excretor negro entre los ciegos (figura 3 ). Las metacercarias midieron $0,89 \mathrm{~mm}(0,78$ a 1,0$)$ de largo $\times 0,48 \mathrm{~mm}(0,4 \mathrm{a}$ $0,54)$ de ancho; el diámetro de la ventosa oral fue $0,14 \mathrm{~mm}(0,12$ a 0,17$)$ y el del acetábulo fue 0,18 $\mathrm{mm}(0,15$ a 0,20$)$. Se depositaron en la colección bajo el código $\mathrm{CCH} 116.111$, lote 837-843.

En lo que respecta a la encuesta previa a los talleres, las maestras aseveraron que los niños no bebían agua de las quebradas por considerarlas contaminadas. Por su parte, los escolares mencionaron mantener un estrecho contacto con ellas, mediante actividades recreativas como nadar y pescar. De otro lado, algunos aceptaron conocer los

Cuadro 1. Macroorganismos recolectados en las veredas La Miel y La Clara de Caldas, Antioquia.

\begin{tabular}{|c|c|c|c|c|}
\hline Vereda & & Especies & Con Paragonimus spp. & $\mathbf{n}$ \\
\hline La Miel & Cangrejos & Hypolobocera bouvieri & 1 & 3 \\
\hline \multirow[t]{4}{*}{ La Clara } & Caracoles & Aroapyrgus colombiensis & 0 & 138 \\
\hline & & Physa acuta & 0 & 17 \\
\hline & & Lymnaea columella & 0 & 57 \\
\hline & Cangrejos & Hypolobocera bouvieri & 0 & 6 \\
\hline
\end{tabular}
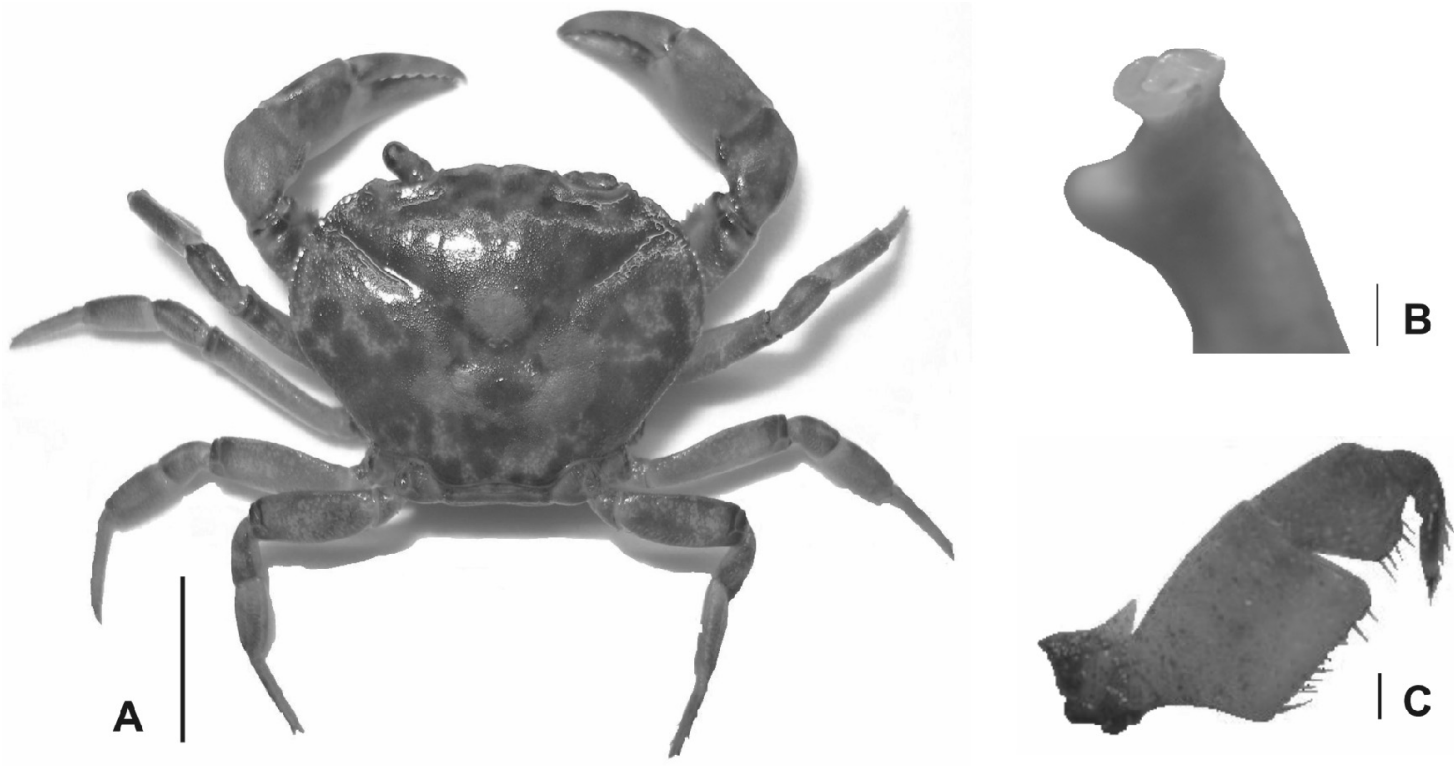

Figura 1. A: Hypolobocera bouvieri; escala: $1 \mathrm{~cm}$. B: Tercer maxilípedo de Hypolobocera bouvieri; escala: $1 \mathrm{~cm}$. C: gonópodo de Hypolobocera bouvieri: escala: $1 \mathrm{~cm}$. Laboratorio de Malacología Médica y Trematodos 


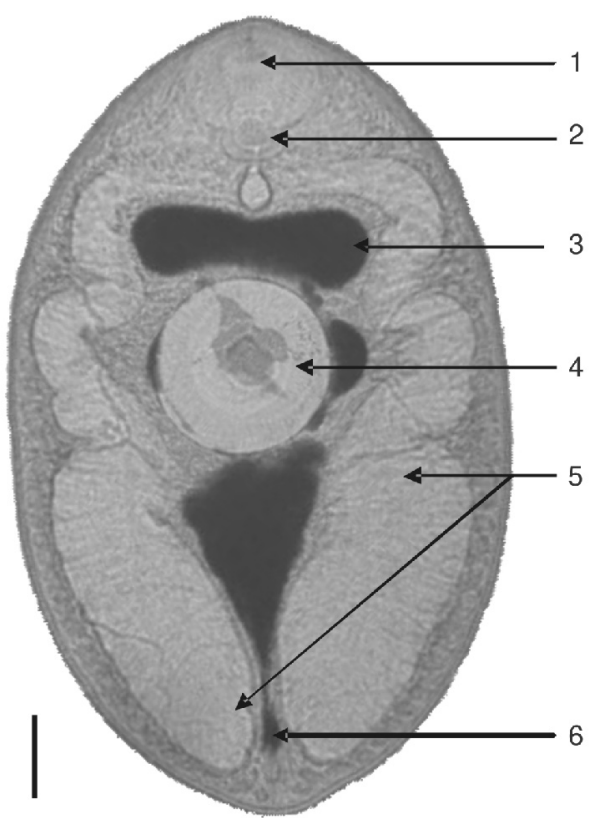

Figura 2. Metacercaria de Paragonimus spp.; escala: $0,1 \mathrm{~mm}$. 1) ventosa oral, 2) faringe, 3) sistema excretor, 4) acetábulo, 5) ciegos intestinales, 6) poro excretor.

Laboratorio de Malacología Médica y Trematodos

animales que habitan en la quebrada, principalmente los peces y los cangrejos, y unos pocos afirmaron haberlos consumido.

En cuanto a los talleres educativos, del primero se destaca el sentido de orientación de todos los participantes al ubicar con facilidad sus viviendas, los lugares para encuentros comunitarios y las zonas de reserva forestal; cabe resaltar que algunos sobresalieron en esta actividad.

Del segundo taller se resalta el concepto que tienen los estudiantes sobre quebrada "limpia": toda quebrada de agua transparente es apta para su uso y, la que no cumpla con esta característica, es "sucia" y peligrosa para la salud. En ambas veredas predominaron por su abundancia las quebradas señaladas como "limpias". En tanto que sólo las quebradas La Miel y La Mina fueron consideradas "sucias". En lo que respecta a su concepto sobre la naturaleza, éste únicamente incluye las "zonas de árboles abundantes" y los animales domésticos.

En el tercer taller, se recolectaron peces, cangrejos y caracoles, y se introdujeron en los acuarios adecuados con los elementos abióticos (cuadro 2). Durante la actividad de captura, los niños jugaron con los peces y les causaron daño; al increparles sobre este hecho, respondieron "los peces no sienten, no lloran ni se quejan como lo hace un perro o un gato".
El acuario en las escuelas fue empleado por las maestras como elemento didáctico de apoyo para la enseñanza de las ciencias naturales e hizo más comprensible la quebrada como ecosistema acuático.

Por medio del cuarto taller, se pudo establecer que los niños conocen los sitios ecoturísticos de las veredas, pero no su importancia medioambiental, es decir, no relacionan la vida silvestre y otros recursos naturales como fundamentales para la supervivencia de su comunidad.

En el quinto taller, gracias a la reflexión generada en el taller anterior, los niños reconocieron como problemáticas medioambientales de su vereda, el vertimiento en la quebrada de basuras, aguas residuales y el lavado de los carros; asimismo, la tala de árboles, la contaminación del aire debido

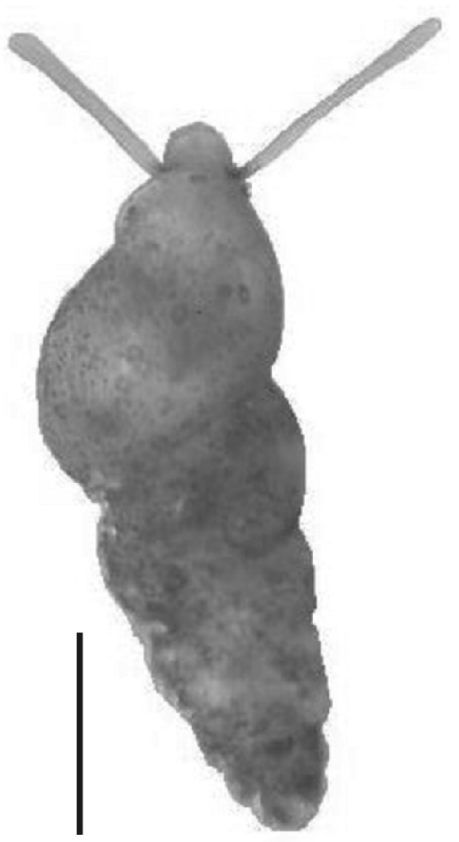

Figura 3. Molusco Aroapyrgus colombiensis; escala: $0,5 \mathrm{~mm}$. Laboratorio de Malacología Médica y Trematodos.

Cuadro 2. Macroorganismos acuáticos recolectados en la quebrada La Miel y La Clara, para la instalación del acuario.

\begin{tabular}{lllr}
\hline Vereda & & Especies & n \\
\hline La Miel & Peces & Guppies: Poecilia reticulata & 30 \\
& Caracoles & Physa acuta & 10 \\
La Clara & Peces & Capitanes, Astroblepidae & 8 \\
& & Sabaletas, Characidae & 15 \\
& & Barbudos, Pygidiidae & 7 \\
& Caracoles & Physa acuta & 11 \\
& & Lymnaea columella & 13 \\
& Cangrejos & Hypolobocera bouvieri & 1 \\
\hline
\end{tabular}


a quemas de basura y los gases que emiten los carros; además, señalaron el maltrato a los animales como una práctica incorrecta. Reconocieron como consecuencia de esto, el deterioro a corto y largo plazo de la salud de las personas y del ecosistema, la muerte y la extinción de especies. Los carteles donde plasmaron las posibles soluciones a los problemas identificados se expusieron en el salón comunal de cada vereda.

\section{Discusión}

Con este trabajo se suma el municipio de Caldas al mapa de distribución de Paragonimus spp. en Antioquia, ante el hallazgo de metacercarias en uno de los cangrejos recolectados en la vereda La Miel; además, se señala esta localidad como el registro del parásito a mayor altitud sobre el nivel del mar en Colombia. A su vez, la vereda La Clara se designa como zona en riesgo para el establecimiento de focos del digéneo al encontrarse todos los huéspedes intermediarios involucrados en el ciclo de vida del parásito, $A$. colombiensis e H. bouvieri.

Estos hechos en el ámbito de la salud pública, sugieren que las estrategias para la prevención y el control de la paragonimosis se realicen en toda la cuenca del río Medellín, mediante propuestas en educación que involucren a la población estudiantil y la comunidad en general, para promover hábitos saludables en relación con los ecosistemas y organismos acuáticos, previniendo el consumo de cangrejos crudos.

Lo anterior surge luego de haber desarrollado esta investigación desde los centros educativos, donde el intercambio de saberes respecto al medio ambiente ayuda a identificar los problemas, y facilita el diseño y realización de las acciones que se implementan hacia la promoción de la salud, siendo una estrategia más eficiente que las medidas impuestas por entes ajenos.

Sin embargo, al realizar actividades con la comunidad es necesario evaluar de manera independiente la percepción de cada una de las fuentes, para evitar la intervención de una sobre la otra. En el caso de la presente investigación se mantuvieron por separado estudiantes y maestras, pues en algunas ocasiones estas últimas trataron de influir sobre la respuesta de los primeros, impidiendo expresar con libertad lo que cada uno percibe.

En esta población estudiantil, es sorprendente que su concepto de naturaleza no incluya la quebrada, ni la fauna silvestre, ni las personas, posiblemente porque la educación ambiental desde su primera infancia, está relacionada con las actividades de explotación de los recursos naturales con fines económicos que los adultos sostienen con los ecosistemas. Por ello, se considera pertinente implementar herramientas educativas, como el acuario, que les permita a docentes, estudiantes y las familias una interacción cercana con los elementos vivos de la quebrada, ayudando a la restauración de los lazos entre las personas y su entorno.

Con esta investigación se corrobora la importancia de abordar el trabajo interdisciplinario y participativo, para promover la salud de las personas, de las comunidades y del entorno.

\section{Agradecimientos}

A la comunidad educativa de las instituciones Monseñor Pedro Luis Álvarez y Ciro Mendía, sede La Clara, del Municipio de Caldas, por su participación permanente en la realización del trabajo; a Iván Darío Vélez, director del PECET, por su apoyo a las investigaciones sobre trematodosis, y al personal del Laboratorio de Malacología Médica y Trematodos, por su acompañamiento.

\section{Conflictos de intereses}

Los autores declaramos que no existen conflictos de intereses en el desarrollo y publicación de este trabajo.

\section{Financiación}

El presente estudio fue evaluado por el Comité para el Desarrollo de la Investigación de la Universidad de Antioquia (CODI), financiado por la Escuela de Microbiología de la Universidad de Antioquia y el Programa de Estudio y Control de Enfermedades Tropicales, PECET.

\section{Referencias}

1. Vélez ID, Ortega JE, Velásquez LE. Paragonimiasis: A view from Columbia. Clin Chest Med. 2002;23:421-31.

2. Botero D, Restrepo M. Parasitosis humanas. Cuarta edición. Medellín: Corporación para Investigaciones Biológicas; 2003.

3. Blair D, Xu ZB, Agatsuma T. Paragonimiasis and the genus Paragonimus. Adv Parasitol. 1999;42:113-222.

4. Vélez I, Velásquez LE, Vélez ID. Morphological description and life cycle of Paragonimus sp. (Trematoda: Troglotrematidae): Causal agent of human paragonimiasis in Colombia. J Parasitol. 2003;89:749-55.

5. MalekE,LittleMD. Aroapyrguscolombiensisn.sp. (Gastropoda: Hydrobiidae), snail intermediate host of Paragonimus caliensis in Colombia. The Nautilus. 1971;85:20-6. 
6. Lamothe-Argumedo R, Malek EA, Meave- Gallegos 0. Aroapyrgus alleei Morrison, (Gastropoda: Hydrobiidae) first intermediate host of Paragonimus mexicanus in Colima, Mexico. J Parasitol. 1983;69:226-8.

7. Uruburu M, Granada M, Velásquez LE. Distribución parcial de Paragonimus (Digenea: Troglotrematidae) en Antioquia, a través de metacercarias en cangrejos dulciacuícolas. Bogotá, D.C. Biomédica. 2008;28;4:562-68.

8. Vélez ID, Velásquez LE. Paragonimosis una investigación multidisciplinaria en salud, biología y cultura en Colombia Medellín: Universidad de Antioquia; 2002.

9. Imperial Collage London. Schistosomiasis control initiative: Advocacy and training interactive guide (CD-ROM). London Wellcome Trust; 2004.

10. Sleigh A. Erradication of schistosomiasis in Guangxi, China. Part 3. Community diagnosis of the worst-affected areas and maintenance strategies for the future. Bull World Health Organ. 1998;76:581-90.

11. Minai M, Hosaka Y, Ohta N. Historical view of schistosomiasis japonica in Japan: Implementation and evaluation of disease- control strategies in Yamanashi prefecture. Parasitol Int. 2003;52:321-6.

12. Instituto Mi Río. Mi Río: guía ecológica y ambiental. Medellín: Instituto Mi Río; 1996. p. 199.

13. Área Metropolitana Valle de Aburrá. Nuestro territorio. Fecha de consulta: 23 de agosto de 2010. Disponible en: http://www.metropol.gov.co/contenidos.php?seccion=14

14. Alcaldía Municipal de Caldas. Caldas con desarrollo y equidad social. Fecha de consulta: 8 de noviembre de 2007. Disponible en: http://www.caldasantioquia.gov.co/ ubicacion.html

15. Campos MR, Rodríguez G. Two new species of freshwater crabs of the genus Hypolobocera from Colombia (Crustacea: Decapoda: Pseudothelphusidae). Proc Biol Soc Wash. 1995;108:649-55.

16. Campos MR. Freshwater crabs from Colombia a taxonomic and distributional study. Bogotá, D.C: Editora Guadalupe Ltda.; 2005. p. 224-94. 